\title{
Platinum-pincer introduction using active ester chemistry
}

\author{
Bart M. J. M. Suijkerbuijk, ${ }^{a}$ Martijn Q. Slagt, ${ }^{a}$ Robertus J. M. Klein Gebbink, ${ }^{a}$ Martin Lutz, ${ }^{b}$ \\ Anthony L. Spek ${ }^{\mathrm{b}}$ and Gerard van Koten ${ }^{\mathrm{a}, *}$ \\ ${ }^{a}$ Debye Institute, Department of Metal-Mediated Synthesis, Utrecht University, Padualaan 8, 3584 CH Utrecht, Netherlands \\ ${ }^{\mathrm{b}}$ Bijvoet Center for Biomolecular Research, Department of Crystal and Structural Chemistry, Utrecht University, Padualaan 8, \\ $3584 \mathrm{CH}$ Utrecht, Netherlands
}

Received 1 May 2002; revised 1 July 2002; accepted 16 July 2002

\begin{abstract}
In search of a new way to attach organometallic pincer complexes to amines via an amide bond, the $N$-hydroxysuccinimide ester of 3,5-bis-[(dimethylamino)methyl]-4-(iodoplatino(II))-benzoic acid was synthesized. This compound reacts with several primary amines to form the corresponding amides cleanly and in high yields. (C) 2002 Elsevier Science Ltd. All rights reserved.
\end{abstract}

Since the first reports on the tridentate ligand $[2,6$ $\left.\left(\mathrm{ECH}_{2}\right)_{2} \mathrm{C}_{6} \mathrm{H}_{3}\right]^{-}\left(\mathrm{E}=\mathrm{NR}_{2}, \mathrm{SR}, \mathrm{PR}_{2}\right)$, now often referred to as 'pincer', 1 a large variety of metals have been incorporated into this chelating unit, providing novel catalysts. ${ }^{2}$ These include catalytically active metals like ruthenium, nickel and palladium, of which the resulting organometallic materials can be applied in hydrogen transfer reactions, ${ }^{3}$ Kharasch additions, ${ }^{4}$ and $\mathrm{C}-\mathrm{C}$ coupling reactions, ${ }^{5}$ respectively. Recently, it was demonstrated that, via platinum incorporation, access can be gained to organometallic $\mathrm{SO}_{2}$-sensors ${ }^{6}$ and biomarkers. ${ }^{7}$ Crucial to the latter application is the availability of easy routes to the para-functionalization of the pincer, eventually in the presence of the metal-carbon bond, in order to perform chemistry beyond the restrictions posed by the relatively small pincer. $^{2}$ Immobilization of the pincer on materials with complementary characteristics seems a promising way to combine the advantages of both. Two distinct approaches for the introduction of metalated pincer moieties via para-functionalization in or onto (organic) scaffolds are possible. Firstly, the organometallic pincer moiety can be introduced by metalation of the pincer ligand after its attachment. This procedure, however, does not ensure a full metalation of the product, especially in the case of a multi-ligand system (e.g. a metallodendrimer). ${ }^{8}$ Secondly, metallated pincers can be introduced directly. This route, however, is not always accessible because of the limited stability of most organometallic fragments under the (harsh) coupling conditions. Recently, we set

\footnotetext{
* Corresponding author. Fax: (+31)30-2523615; e-mail: g.vankoten@ chem.uu.nl
}

out to develop a gentle coupling chemistry for the attachment of the pincer moiety, with retention of the C-Pt bond, in order to attach it also to chemically less inert molecules.

One of the most stable heteroatomic bonds known to organic chemists is the amide bond. This bond is accessible via several synthetic routes, one of the most gentle being the activation of a carboxylic acid (i.e. synthesis of an activated ester) followed by reaction with an amine. Here, we describe the synthesis of such an 'activated pincer'-Pt $\mathbf{1}$ and its coupling chemistry with various amines.

Activated platinum-pincer $\mathbf{1}$ was synthesized according to the route depicted in Scheme 1. Bromo-iodo-compound $\mathbf{2}$ was obtained by a seven-step synthesis, starting from 3,5-dimethylaniline in $36 \%$ overall yield. ${ }^{9}$ Platinum incorporation was accomplished via oxidative addition of 2 to $\left[\mathrm{Pt}(p \text {-tol })_{2}\left(\mathrm{Et}_{2} \mathrm{~S}\right)\right]_{2}$ using Canty's procedure, ${ }^{10}$ which yielded $80 \%$ of $\mathbf{3}$ as a white, crystalline solid. The carboxylic acid derivative $\mathbf{4}$ was obtained by lithiation via exchange with the iodine and subsequent quenching of the resulting bimetallic species with $\mathrm{CO}_{2}{ }^{11}$ In order to overcome halide-scrambling during this reaction, the resulting solid was treated with $\mathrm{NaI}$ in acetone, yielding $78 \%$ of $\mathbf{4}$ as a light yellow solid. ${ }^{11}$ Making use of $N, N^{\prime}$-dicyclohexylcarbodiimide (DCC) as a coupling agent, activated pincer 1 was synthesized from 4 and $N$-hydroxysuccinimide (NHS) with pyridine as a base to give a light yellow solid (98\%). ${ }^{12,13}$ Spectroscopic evidence for the formation of 1 includes low field shifts for the aromatic protons $(7.58 \mathrm{ppm})$ compared to 


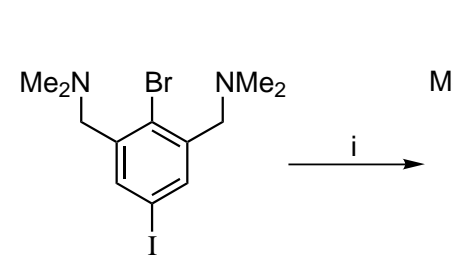

2<smiles>CC1CN(C)Cc2cc(I)cc(Br)c21</smiles>

3<smiles>CN1Cc2cc(C(=O)O)cc(I)c2N1C</smiles>

4

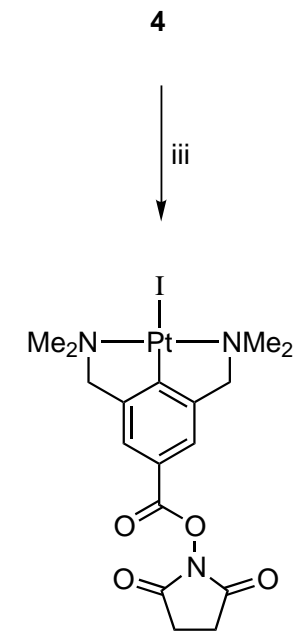

1

Scheme 1. Reagents and conditions: (i) $\left[\mathrm{Pt}(p \text {-tol })_{2}\left(\mathrm{Et}_{2} \mathrm{~S}\right)\right]_{2}, \mathrm{C}_{6} \mathrm{H}_{6}, 2 \mathrm{~h}$, reflux; (ii) (1) $t$ - $\mathrm{BuLi}, \mathrm{THF}, 2 \mathrm{~min},-100^{\circ} \mathrm{C},(2) \mathrm{CO}_{2(\mathrm{~g})}, 1 \mathrm{~h}$, rt, (3) NaI, acetone, $1 \mathrm{~h}$, rt; (iii) NHS, DCC, pyridine, THF, 16 h, rt.

the parent compound 4 (7.51 ppm). To unequivocally prove that 1 was obtained, crystals were grown by slowly diffusing $\mathrm{Et}_{2} \mathrm{O}$ into a concentrated solution of $\mathbf{1}$ in methylene chloride. The crystal structure is shown in Fig. $1 .^{14-17}$

The scope and reactivity of $\mathbf{1}$ toward several types of amines were investigated (Scheme 2). ${ }^{18}$ First, 1 was reacted with $n$-butylamine by stirring both reagents in

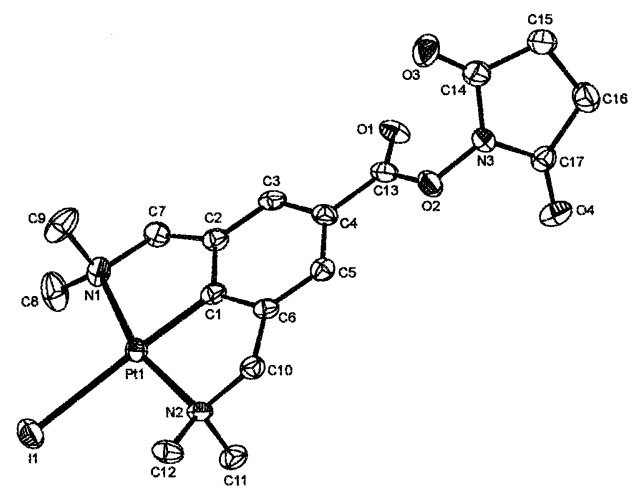

Figure 1. Displacement ellipsoid plot (50\% probability) of $\mathbf{1}$. Hydrogen atoms have been omitted for clarity. Selected bond lengths $(\AA)$, angles and torsion angles $\left(^{\circ}\right)$ : Pt1-C1 1.923(5), Pt1-N1 2.108(4), Pt1-N2 2.102(4), Pt1-I1 2.7135(4), C4-C13 1.475(6), C13-O1 1.192(6), C13-O2 1.412(6), O2-N3 1.390(5), I1-Pt1-C1 177.83(13), N1-Pt1-N2 162.89(15), C1-Pt1N1-C7 23.4(3), C1-Pt1-N2-C10 23.3(3), C3-C4-C13-O1 $-9.4(7), \mathrm{C} 13-\mathrm{O} 2-\mathrm{N} 3-\mathrm{C} 14$ 74.0(5).
$\mathrm{CH}_{2} \mathrm{Cl}_{2}$ at room temperature for 16 hours. After a basic work-up, the product was precipitated from a $\mathrm{CH}_{2} \mathrm{Cl}_{2}$ solution with $\mathrm{Et}_{2} \mathrm{O}$, yielding $88 \%$ of $\mathbf{5}$, without the need for additional purification steps. The formation of $\mathbf{5}$ was clearly indicated by the appearance of NMR resonances at $\delta=7.48 \mathrm{ppm}$ (br t, $\mathrm{NHC}(\mathrm{O})$ ) and $\delta=3.36$ ppm (q, $\left.\mathrm{CH}_{2} \mathrm{NHC}(\mathrm{O})\right)$ and the change of the chemical shift of the aromatic protons from $\delta=7.58 \mathrm{ppm}$ to $\delta=7.36 \mathrm{ppm}$. Applying the same procedure, 6 was synthesized using 4-methylbenzylamine as the amine. After work-up 6 was obtained in an $80 \%$ yield as a white solid (new NMR resonances at $\delta=6.22 \mathrm{ppm}$ (br $\mathrm{t}, \mathrm{NHC}(\mathrm{O}))$ and $\delta=4.56 \mathrm{ppm}\left(\mathrm{d}, \mathrm{CH}_{2} \mathrm{NHC}(\mathrm{O})\right)$, aromatic protons at $\delta=7.28)$. $\quad N^{\alpha}$-Boc-L-lysine was attached to the platinum pincer moiety following the procedure applied by Tampé and coworkers. ${ }^{19}$ First, Boc-protected lysine was treated with chlorotrimethylsilane to enhance its solubility in the reaction medium. Next, 1 was added, the reaction mixture was stirred for 16 hours and after purification, including precipitation, a white solid 7 was obtained in 56\% yield (new NMR resonances at $\delta=7.58 \mathrm{ppm}$ (br t, $\mathrm{N} H \mathrm{C}(\mathrm{O})$ ) and $\delta=3.38$ $\operatorname{ppm}\left(\mathrm{q}, \mathrm{CH}_{2} \mathrm{NHC}(\mathrm{O})\right)$, aromatic protons at $\left.\delta=7.38\right)$. Activated pincer 1 was shown not to react with less reactive amines like $p$-toluidine and diethylamine.

In conclusion, an organometallic active ester was synthesized and was shown to cleanly react with primary amines. The results are promising with regard to direct incorporation of metalated (organometallic platinum) pincer fragments in or onto large organic frameworks 

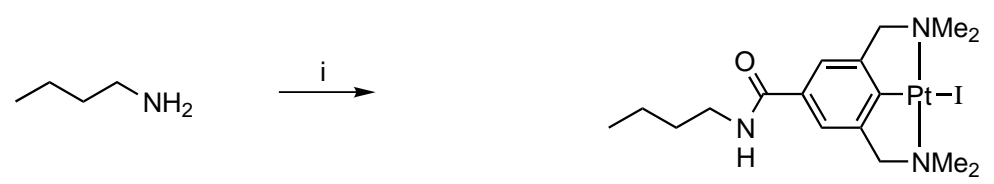

5<smiles>Cc1ccc(CN)cc1</smiles>

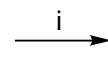<smiles>CC(C)(C)OC(=O)N[C@@H](CCCCN)C(=O)O</smiles>

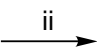

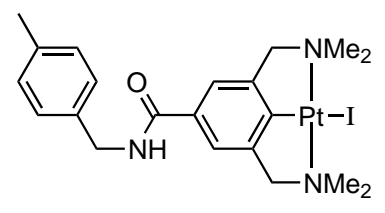

6

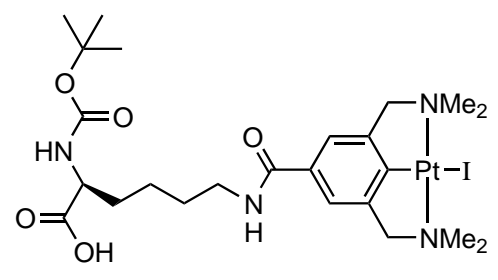

7

Scheme 2. Reagents and conditions: (i) 1, $\mathrm{CH}_{2} \mathrm{Cl}_{2}, 16 \mathrm{~h}$, rt; (ii) (a) $\mathrm{TMSCl}, \mathrm{Et}_{3} \mathrm{~N}, \mathrm{CH}_{2} \mathrm{Cl}_{2}, 1 \mathrm{~h}$, reflux; (b) 1, 16 h, rt.

(i.e. without stepwise ligand incorporation followed by metalation). The reactivity of $\mathbf{1}$ towards other amines is currently under investigation. Furthermore, the incorporation of metals other than platinum into the activated pincer ligand is being pursued.

\section{Acknowledgements}

This work was supported by The Netherlands Foundation for Chemical Sciences (CW) with financial aid from the Netherlands Organization for Scientific Research (NWO) (M.L. and A.L.S.) and STW (M.Q.S.) and by the Netherlands Research School Combination Catalysis (NRSC-C) (R.J.M.K.G.). Anca van der Kerk-van Hoof (Mass Spectrom. Dept., UU) is gratefully acknowledged for the electrospray mass spectrometric studies.

\section{References}

1. (a) Moulton, C. J.; Shaw, B. L. J. Chem. Soc., Dalton Trans. 1976, 1020-1024; (b) van Koten, G.; Timmer, K.; Noltes, J. G.; Spek, A. L. J. Chem. Soc., Chem. Commun. 1978, 250-252; (c) van Koten, G.; Jastrzebski, J. T. B. H.; Noltes, J. G. J. Organomet. Chem. 1978, 148, 233-245; (d) Creaser, C. S.; Kaska, W. C. Inorg. Chim. Acta 1978, 30, L325-L326.

2. Albrecht, M.; van Koten, G. Angew. Chem., Int. Ed. 2001, 40, 3750-3781.
3. Dani, P.; Karlen, T.; Gossage, R. A.; Gladiali, S.; van Koten, G. Angew. Chem., Int. Ed. 2000, 39, 743-745.

4. Gossage, R. A.; van de Kuil, L. A.; van Koten, G. Acc. Chem. Res. 1998, 31, 423-431.

5. Dijkstra, H. P.; Meijer, M. D.; Patel, J.; Kreiter, R.; van Klink, G. P. M.; Lutz, M.; Spek, A. L.; Canty, A. J.; van Koten, G. Organometallics 2001, 20, 3159-3168.

6. Albrecht, M.; Lutz, M.; Spek, A. L.; van Koten, G. Nature 2000, 406, 970-974.

7. Albrecht, M.; Rodríguez, G.; Schoenmaker, J.; van Koten, G. Org. Lett. 2000, 22, 3461-3464.

8. Recently, however, transcyclometalation processes were shown to be an improved method for full metal incorporation after ligand introduction: Dijkstra, H. P.; Albrecht, M.; van Koten, G. Chem. Commun. 2002, 126-127.

9. Rodríguez, G.; Albrecht, M.; Schoenmaker, J.; Ford, A.; Lutz, M.; Spek, A. L.; van Koten, G. J. Am. Chem. Soc. 2002, 124, 5127-5138.

10. Canty, A. J.; Patel, J.; Skelton, B. W.; White, A. H. J. Organomet. Chem. 2000, 599, 195-199.

11. This compound was prepared according to a procedure, we developed earlier. See: Slagt, M. Q.; Klein Gebbink, R. J. M.; Lutz, M.; Spek, A. L.; van Koten, G. J. Chem. Soc., Dalton Trans. 2002, 2591-2592. To a cooled solution $\left(-100^{\circ} \mathrm{C}\right)$ of $3(2.66 \mathrm{~g}, 4.50 \mathrm{mmol})$ in dry THF $(150$ $\mathrm{mL})$ was added ${ }^{t} \mathrm{BuLi}(6.00 \mathrm{~mL}$ of a $1.5 \mathrm{M}$ solution in pentane, $9.00 \mathrm{mmol}$ ) and the resulting yellow solution was stirred for 2 min. Next, dry $\mathrm{CO}_{2}$ was bubbled through, upon which the solution became turbid (white), and the suspension was then allowed to reach room temperature. $\mathrm{H}_{2} \mathrm{O}(1 \mathrm{~mL})$ was subsequently added whereupon the precipitate dissolved. All volatiles were removed 
in vacuo, the residue was taken up in $\mathrm{CHCl}_{3}(200 \mathrm{~mL})$ and washed with saturated $\mathrm{NH}_{4} \mathrm{Cl}$ solution $(2 \times 10 \mathrm{~mL})$. The organic layer was dried $\left(\mathrm{MgSO}_{4}\right)$ and the volatiles were removed. Next, the remaining solid was redissolved in acetone and treated with $\mathrm{NaI}(1.20 \mathrm{~g}, 8.00 \mathrm{mmol})$ and the yellow solution was stirred for $1 \mathrm{~h}$. The solution was filtered over Celite and the volatiles were evaporated. The residue was redissolved in $\mathrm{CHCl}_{3}(200 \mathrm{~mL})$ and washed with $\mathrm{H}_{2} \mathrm{O}(10 \mathrm{~mL})$, the organic layer was dried $\left(\mathrm{MgSO}_{4}\right)$ and concentrated to $10 \mathrm{~mL}$. $\mathrm{Et}_{2} \mathrm{O}$ was added to induce precipitation. The light yellow product was collected by centrifugation. Yield: $1.96 \mathrm{~g}(78 \%) .{ }^{1} \mathrm{H}$ NMR (DMSO- $\left.d_{6}\right)$ $\delta=7.41(\mathrm{~s}, 2 \mathrm{H}, \operatorname{Ar} H) ; 4.13\left(\mathrm{~s},{ }^{3} J_{\mathrm{PtH}}=39.0 \mathrm{~Hz}, 4 \mathrm{H}, \mathrm{CH}_{2}\right)$; $3.07\left(\mathrm{~s},{ }^{3} J_{\mathrm{PtH}}=28.8 \mathrm{~Hz}, 12 \mathrm{H}, \mathrm{N}\left(\mathrm{CH}_{3}\right)_{2}\right) \mathrm{ppm} .{ }^{13} \mathrm{C}\left\{{ }^{1} \mathrm{H}\right\}$ NMR (DMSO- $d_{6}$ ) $\delta=168.2,156.0,144.1,125.6,120.6$, 75.4, $55.6 \mathrm{ppm}$.

12. (a) Garbiras, B. J.; Marburg, S. Synthesis 1999, 270-274; (b) Leikauf, E.; Barnekow, F.; Köster, H. Tetrahedron 1995, 51, 3793-3802.

13. To a solution of $4(538 \mathrm{mg}, 0.97 \mathrm{mmol})$ in dry THF (50 $\mathrm{mL}$ ) were added $N$-hydroxysuccinimide (112 $\mathrm{mg}, 0.98$ mmol), DCC (202 mg, $0.98 \mathrm{mmol}$ ), and dry pyridine (1 $\mathrm{mL}$ ). The clear yellow solution was stirred for $16 \mathrm{~h}$ and a white precipitate (dicyclohexylurea) was then filtered off. The solvent was evaporated and the remaining solid was redissolved in $\mathrm{CH}_{2} \mathrm{Cl}_{2}(5 \mathrm{~mL})$ and a white precipitate was filtered off again. This cycle of dissolving the solid in $\mathrm{CH}_{2} \mathrm{Cl}_{2}$ and filtering off the white precipitate was repeated until no more white precipitate was detected. After evaporation of the solvent in vacuo, the yellow solid was taken up in $\mathrm{CH}_{2} \mathrm{Cl}_{2}(5 \mathrm{~mL})$ and $\mathrm{Et}_{2} \mathrm{O}$ was added to induce precipitation. The light yellow product was then collected by centrifugation. Further purification of 1 can be achieved by trituration with acetone. Yield: $613 \mathrm{mg}(97 \%)$, after trituration $408 \mathrm{mg}(65 \%) .{ }^{1} \mathrm{H}$ NMR $\left(\mathrm{CDCl}_{3}\right) \delta=7.61(\mathrm{~s}, 2 \mathrm{H}, \mathrm{Ar} H) ; 4.06\left(\mathrm{~s},{ }^{3} J_{\mathrm{PtH}}=45.9 \mathrm{~Hz}\right.$, $\left.4 \mathrm{H}, \mathrm{ArCH}_{2}\right) ; 3.17\left(\mathrm{~s},{ }^{3} \mathrm{~J}_{\mathrm{PtH}}=39.9 \mathrm{~Hz}, 12 \mathrm{H}, \mathrm{N}\left(\mathrm{CH}_{3}\right)_{2}\right)$; $2.89\left(\mathrm{~s}, 4 \mathrm{H}, \mathrm{CH} \mathrm{H}_{2} \mathrm{C}(\mathrm{O})\right)$ ppm. ${ }^{13} \mathrm{C}\left\{{ }^{1} \mathrm{H}\right\}$ NMR $\left(\mathrm{CDCl}_{3}\right)$ $\delta=169.5,162.5,160.2,144.2,122.0,119.8,76.3,56.3$, $25.7 \mathrm{ppm}$. Note: 1 undergoes halogen-exchange in chloroform. Elem. anal. found (calcd) for $\mathrm{C}_{17} \mathrm{H}_{22} \mathrm{IN}_{3} \mathrm{O}_{4} \mathrm{Pt}$ : C, 31.20 (31.09); H, 3.30 (3.45); N, 6.42 (6.31). ESI MS $m / z$ $676.9\left([M+\mathrm{Na}]^{+}\right), 527.3\left([M-\mathrm{I}]^{+}\right)$.

14. Crystal structure determination of $1 . \mathrm{C}_{17} \mathrm{H}_{22} \mathrm{IN}_{3} \mathrm{O}_{4} \mathrm{Pt}$, $M_{\mathrm{r}}=654.37$, yellow plate, $0.12 \times 0.12 \times 0.03 \mathrm{~mm}^{3}, T=$ $125(2) \mathrm{K}$, monoclinic, space group $P 2_{1} / c$ (No. 14), $a=$ 6.1163(1), $\quad b=23.3240(4), \quad c=14.4823(2) \quad \AA$, $\beta=106.4139(7)^{\circ}, \quad V=1981.80(5) \AA^{3} . Z=4, \rho=2.193 \mathrm{~g} /$ $\mathrm{cm}^{3}$. X-ray intensities were measured on a Nonius Kappa CCD diffractometer with rotating anode $(\lambda=0.71073 \AA)$. Analytical absorption correction $\left(\mu=8.66 \mathrm{~mm}^{-1}, 0.33\right.$ 0.55 transmission). 27,598 measured reflections up to $(\sin \theta / \lambda)_{\max }=0.65 \AA^{-1}$, of which 4524 were unique $\left(R_{\text {int }}=\right.$ $0.0459)$. The structure was solved with automated Patterson methods (DIRDIF97 ${ }^{15}$ ) and refined with SHELXL97 ${ }^{16}$ against $F^{2}$ of all reflections. $R[I>2 \sigma(I)]$ : $R_{1}=0.0275, w R_{2}=0.0614 . R$ [all reflections]: $R_{1}=0.0359$, $w R_{2}=0.0648 . \quad S=1.020$. Residual electron density between -1.13 and $2.90 \mathrm{e} / \AA^{3}$. Molecular illustration, structure checking and calculations were performed with the PLATON package. ${ }^{17}$

15. Beurskens, P. T.; Admiraal, G.; Beurskens, G.; Bosman, W. P.; Garcia-Granda, S.; Gould, R. O.; Smits, J. M. M.; Smykalla, C. The DIRDIF97 program system, Technical Report of the Crystallography Laboratory; University of Nijmegen: The Netherlands, 1997.

16. Sheldrick, G. M. SHELXL-97. Program for crystal structure refinement; University of Göttingen, Germany, 1997.

17. Spek, A. L. PLATON, a multipurpose crystallographic tool; Utrecht University: The Netherlands, 2001.

18. Spectroscopic analyses of amides:

5: White crystalline solid. ${ }^{1} \mathrm{H}$ NMR (acetone- $d_{6}$ ) $\delta=7.48$ (br t, $1 \mathrm{H}, \mathrm{NH}) ; 7.36(\mathrm{~s}, 2 \mathrm{H}, \mathrm{Ar} H) ; 4.14\left(\mathrm{~s},{ }^{3} J_{\mathrm{PtH}}=45.6\right.$ $\left.\mathrm{Hz}, 4 \mathrm{H}, \mathrm{ArCH}_{2}\right) ; 3.36\left(\mathrm{q},{ }^{3} J_{\mathrm{HH}}=6.6 \mathrm{~Hz}, 2 \mathrm{H}, \mathrm{CH}_{2} \mathrm{NH}\right)$; $3.15\left(\mathrm{~s},{ }^{3} J_{\mathrm{PtH}}=39.3 \mathrm{~Hz}, 12 \mathrm{H}, \mathrm{N}\left(\mathrm{CH}_{3}\right)_{2}\right) ; 1.57$ (qui, ${ }^{3} J_{\mathrm{HH}}=$ $\left.6.6 \mathrm{~Hz}, 2 \mathrm{H}, \mathrm{CH}_{2} \mathrm{CH}_{2} \mathrm{NH}\right) ; 1.38\left(\mathrm{sex},{ }^{3} \mathrm{~J}_{\mathrm{HH}}=6.6 \mathrm{~Hz}, 2 \mathrm{H}\right.$, $\left.\mathrm{CH}_{2} \mathrm{CH}_{2} \mathrm{CH}_{2} \mathrm{NH}\right) ; 0.92\left(\mathrm{t},{ }^{3} J_{\mathrm{HH}}=6.6 \mathrm{~Hz}, 3 \mathrm{H}, \mathrm{CH}_{2} \mathrm{CH}_{3}\right)$ ppm. ${ }^{13} \mathrm{C}\left\{{ }^{1} \mathrm{H}\right\}$ NMR (acetone- $\left.d_{6}\right) \delta=168.1,154.7,144.8$, 131.5, 119.2, 77.1, 56.5, 40.0, 32.7, 20.8, 14.1 ppm. Elem. anal. found (calcd) for $\mathrm{C}_{17} \mathrm{H}_{28} \mathrm{IN}_{3} \mathrm{OPt}$ : C, 33.30 (33.34); H, 4.66 (4.61); N, 6.81 (6.86).

6: White solid. ${ }^{1} \mathrm{H}$ NMR $\left(\mathrm{CDCl}_{3}\right) \delta=7.28$ (s, 2H, $\left.\mathrm{Ar} H\right)$; $7.24\left(\mathrm{~d},{ }^{3} J_{\mathrm{HH}}=8.1 \mathrm{~Hz}, 2 \mathrm{H}, \operatorname{Ar} H\right) ; 7.16\left(\mathrm{~d},{ }^{3} J_{\mathrm{HH}}=8.1 \mathrm{~Hz}\right.$, $2 \mathrm{H}, \operatorname{Ar} H) ; 6.22($ br t, $1 \mathrm{H}, \mathrm{NH}) ; 4.56\left(\mathrm{~d},{ }^{3} J_{\mathrm{HH}}=5.4 \mathrm{~Hz}\right.$, $\left.2 \mathrm{H}, \mathrm{CH}_{2} \mathrm{NH}\right) ; 4.03\left(\mathrm{~s},{ }^{3} \mathrm{~J}_{\mathrm{PtH}}=45.9 \mathrm{~Hz}, 4 \mathrm{H}, \mathrm{ArCH}_{2}\right) ; 3.17$ $\left(\mathrm{s},{ }^{3} J_{\mathrm{PtH}}=38.7 \mathrm{~Hz}, 12 \mathrm{H}, \mathrm{N}\left(\mathrm{CH}_{3}\right)_{2}\right) ; 2.34\left(\mathrm{~s}, 3 \mathrm{H}, \mathrm{ArCH}_{3}\right)$ ppm. ${ }^{13} \mathrm{C}\left\{{ }^{1} \mathrm{H}\right\}$ NMR $\left(\mathrm{CDCl}_{3}\right) \quad \delta=168.0,154.7,143.7$, 137.3, 135.3, 130.0, 129.4, 127.9, 118.4, 76.2, 56.3, 43.9, $21.1 \mathrm{ppm}$. Elem. anal. found (calcd) for $\mathrm{C}_{21} \mathrm{H}_{28} \mathrm{IN}_{3} \mathrm{OPt}$ : C, 38.26 (38.19); H, 4.34 (4.27); N, 6.31 (6.36).

7: Off-white solid. ${ }^{1} \mathrm{H}$ NMR (acetone- $\left.d_{6}\right) \delta=7.58(\mathrm{br}, 1 \mathrm{H}$, $\left.\mathrm{CH}_{2} \mathrm{NH}\right) ; 7.38(\mathrm{~s}, 2 \mathrm{H}, \mathrm{Ar} H) ; 6.16\left(\mathrm{~d},{ }^{3} J_{\mathrm{HH}}=8.4 \mathrm{~Hz}, 1 \mathrm{H}\right.$, $\left.\mathrm{CHNHC}(\mathrm{O}) \mathrm{O}^{t} \mathrm{Bu}\right) ; 4.14\left(\mathrm{~s},{ }^{3} J_{\mathrm{PtH}}=44.7 \mathrm{~Hz}, 4 \mathrm{H}, \mathrm{ArCH}_{2}\right.$ overlapping q, $1 \mathrm{H}, \mathrm{NHCHCOOH}) ; 3.38$ (q, ${ }^{3} J_{\mathrm{HH}}=6.6$ $\mathrm{Hz}, 2 \mathrm{H}, \mathrm{CH}_{2} \mathrm{NHC}(\mathrm{O})$ ); $3.15\left(\mathrm{~s},{ }^{3} \mathrm{~J}_{\mathrm{PtH}}=39.0 \mathrm{~Hz}, 12 \mathrm{H}\right.$, $\left.\mathrm{N}\left(\mathrm{CH}_{3}\right)_{2}\right) ; 1.80\left(\mathrm{~m}, 2 \mathrm{H}, \mathrm{CH}_{2} \mathrm{CH}(\mathrm{C}(\mathrm{O}))(\mathrm{NH})\right) ; 1.62(\mathrm{~m}$, $2 \mathrm{H}, \mathrm{CH}_{2} \mathrm{CH}_{2} \mathrm{CH}(\mathrm{C}(\mathrm{O}))(\mathrm{NH})$ ); 1.51 (qui, $2 \mathrm{H}, 8.0 \mathrm{~Hz}$, $\mathrm{CH}_{2} \mathrm{CH}_{2} \mathrm{NHC}(\mathrm{O})$ ); 1.39 (s, 9H, Boc) ppm. ${ }^{13} \mathrm{C}\left\{{ }^{1} \mathrm{H}\right\}$ NMR $\left(\mathrm{CDCl}_{3}\right) \quad \delta=174.3,168.3,156.6,154.8,144.8$, 131.4, 119.2, 79.1, 77.1, 56.5, 54.3, 39.9, 32.2, 30.1, 28.6, $23.9 \mathrm{ppm}$. Elem. anal. found (calcd) for $\mathrm{C}_{24} \mathrm{H}_{39} \mathrm{IN}_{4} \mathrm{O}_{5} \mathrm{Pt}$ : C, 36.83 (36.69); H, 5.11 (5.00); N, 10.05 (10.19).

19. Schmitt, L.; Dietrich, C.; Tampé, R. J. Am. Chem. Soc. 1994, 116, 8485-8491. 